\section{Drawing Chemical Structures}

\section{by Jeffery Leigh}

Although the drawing of chemical structures is not strictly a nomenclature matter as usually understood, it is a way of conveying the structure of a chemical compound just as is an IUPAC systematic name, though using a visual language rather than a verbal one. Consequently, it is necessary to use certain widely-accepted conventions when drawing a chemical structure, particularly if that structure is threedimensional and the representation of that structure as drawn on a sheet of paper is necessarily in two dimensions. IUPAC has attempted to define preferable methods for achieving this, and the new edition of Principles, unlike its predecessor, contains a summary of what is currently considered to be best practice to this end.

Certain recommendations are almost self-evident. For example, it is common, but not mandatory, to use the same font and font size in your structural diagrams as in your text. Principles uses Times New Roman, which was this editor's choice. Some people prefer to use sans serif fonts such as Arial, though this can lead to minor confusion between symbols, such as 1 and 1(Times New Roman) with I and 1(Arial). Unusual abbreviations used in a diagram label should be defined somewhere in the article being written. It is not enough to assume that everyone will know what thf stands for, though this is probably acceptable for Ph. Bond lengths, thicknesses, and angles should be used consistently in all your diagrams. You should decide whether to represent aromatic rings as localized systems, as in (a) below, or as delocalized systems as in (b). Which you choose is not important, as long as your symbolism is clearly understood and is used consistently. (a)

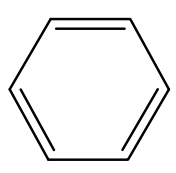

(b)

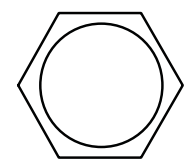

In the past, a variety of methods has been employed to portray a three-dimensional structure in two dimensions, for example, to show whether a bond which does not lie in the plane of the paper is pointing behind that plane or forwards towards the reader. In
Principles, we have settled for conventional methods, as shown below in (i) for the tetrahedral molecule $\mathrm{CH}_{4}$.

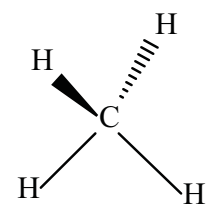

(i)

\section{bond pointing in front of plane of pape

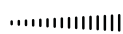 bond pointing behind plane of paper}

bond in plane of paper
Principles also introduces adaptations of this convention, for example, in order to represent certain ring structures, as in the representation for the ferrocene molecule shown below (ii).

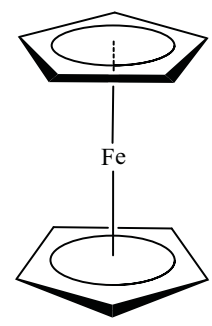

More complicated polyhedral shapes than tetrahedral are frequently encountered in chemistry, and these are also discussed in Principles, which introduces the most common three-dimensional structures found in coordination chemistry and also the most common projections used to represent the three-dimensional structures of organic molecules. For the beginner, these are sometimes not easy to understand.

The octahedron is a shape very often found in coordination chemistry, but the manner in which it is represented depends upon the circumstances. In the representation (iii) below of a coordination complex formally written as [Mabcdef], the bonds between the ligands ( $a, b, c$, etc.) to the central metal [not specifically indicated in diagrams (iii)-(v)] are represented using the formalism described above in (iii), but in (iv) the principal plane of the octahedron is drawn, but only the bonds to ligands e and f. Finally, in ( $v$ ), only the octahedron is delineated and no bonds. The edges of the solid octahedron invisible to a viewer are represented by dashed lines. Yet, all three are acceptable representations of the molecule [Mabcdef], and should be equally comprehensible to the informed reader.

Organic chemists have related problems when representing organic structures in three dimensions, and they use a variety of projections to do this, the prin- 


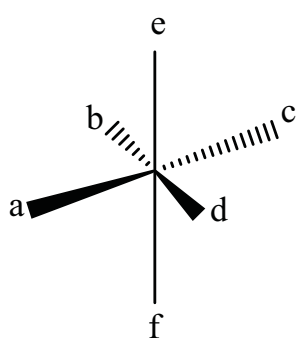

(iii)

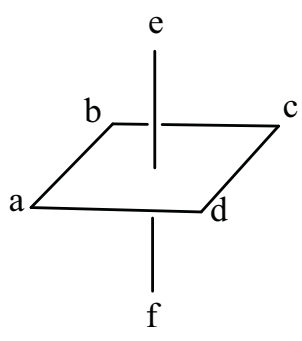

(iv)

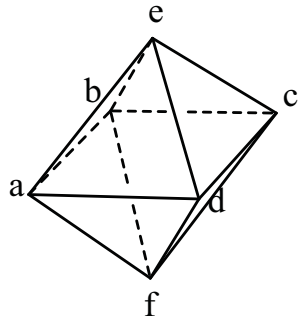

(v) cipal ones being named after their inventors: Fischer, Haworth, and Newman. These particular projections are usually applied to specific classes of molecule. In a Fischer projection, the bonds to the carbon at the center of the tetrahedron are not represented as in the drawing of Cabcd (vi), but in a plane as in (vii), the convention being that bonds drawn vertically are pointing behind the plane of the paper, and the horizontal ones in front. The central carbon atom is not specifically represented. This type of projection is used primarily for carbohydrates and amino acids.

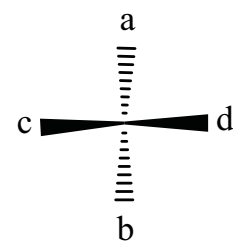

(vi)

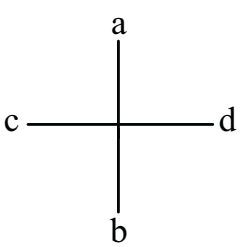

(vii)
A Newman projection is employed to represent more complex molecules, such as an ethane-type molecule $\mathrm{C}_{2}$ abcdef, where different conformations with respect to a selected carbon-carbon bond may be pres- ent. This is illustrated in (viii) and (ix) below. Finally, Haworth projections are often applied to compounds such as monosaccharides and polysaccharides.

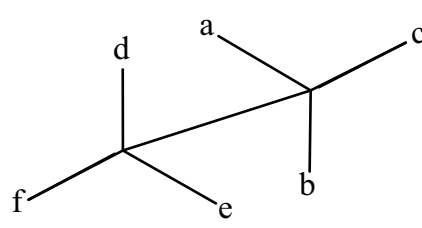

(viii)

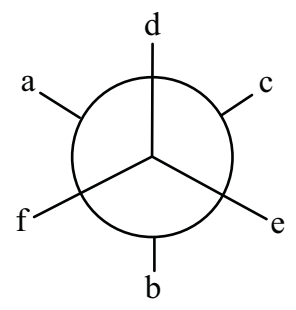

(ix)
The use of all these devices and more, including various ways to represent conformations, are discussed in the new volume of Principles, together with appropriate examples and literature references.

Jeffery Leigh is the editor and contributing author of Principles of Chemical Nomenclature-A Guide to IUPAC Recommendations, 2011 Edition (RSC 2011, ISBN 978-1-84973-007-5). Leigh is emeritus professor at the University of Sussex and has been active in IUPAC nomenclature since 1973.

www.iupac.org/publications/ci/indexes/nomenclature-notes.html

\section{continued from page 25}

which discusses membranes used for water purification, provides a good overview of existing materials and developments.

Glass is not a new material. Nevertheless, new technologies open new opportunities for sustainability, as shown in chapter 20. Examples are thermo-chrome and photo-chrome functionalities and self-cleaning properties. Cost effective solutions must be developed.

The book is well illustrated and chapters are well documented with up to 150 references. Unfortunately, not all figures and tables reveal the data source. The subject index is large and allows easy access to all topics.
The book is valuable in more than one way. It covers a wide field of materials. For a given subject the reader finds extensive data. With a look at the conclusions he or she will get an understanding of what we can do to become more sustainable. It is a large book and not cheap. But the density of information and the different views it provides on an important subject make it worth the money. I thank and congratulate Trevor Letcher and Janet Scott for their effort in publishing this book.

\section{www.rsc.org/shop/books/2012/9781849734073.asp}

